\title{
Ventana torácica en el tratamiento del empiema pleural crónico en el Hospital Nacional Cayetano Heredia, 1990-1998
}

\author{
Maekawa Ykehara, Rosalba Lupe; Amez Olivera, Jessica Mercedes y Castañeda \\ Saldaña Enrique* \\ Profesor asociado de Cirugía, Departamento de Cirugía, Universidad Peruana Cayetano \\ Heredia. Jefe del Servicio de Cirugía de Tórax y Cardiovascular, Hospital Nacional Cayetano \\ Heredia.
}

\section{RESUMEN}

Objetivo: Describir los resultados de la ventana torácica en el manejo del empiema pleural. Pacientes y métodos: Estudio descriptivo, retrospectivo y transversal. Se incluyeron pacientes sometidos a ventana torácica (toracostomía) entre 1990-1998, en el Hospital Nacional Cayetano Heredia. Se estudió el tratamiento quirúrgico (técnica, hallazgos y complicaciones), morbilidad y mortalidad. De los 59 casos estudiados 39 (66.1\%) fueron varones y $20(33.9 \%)$ mujeres. La edad promedio fue $30.9 \pm 14$ años. Resultados: Según la etiología, 55.93\% tuvieron empiema pleural tuberculoso, 28.81\% empiema post cirugía pulmonar, $11.86 \%$ empiema post neumónico y 3.39\% empiema por traumatismo. El $95 \%$ de los casos fueron previamente drenados con tubo de toracostomía. El patrón radiológico predominante fue el hidroneumotórax (58\%). El 64.4\% fueron intervenidos bajo anestesia regional. El tiempo promedio operatorio fue de $57.3 \pm 28$ minutos. Los arcos costales resecados con más frecuencia fueron el $6^{\circ}-7^{\circ}(25 \%)$. El tiempo promedio de hospitalización fue $38.6 \pm 26.9$ días, el tiempo promedio postoperatorio fue $8.5 \pm 8.3$ días. De los pacientes que tuvieron un seguimiento mayor a 3 meses (33 pacientes), se pudo confirmar el cierre de la ventana torácica en 13 casos y reexpansión pulmonar en 21 casos. Conclusiones: La indicación principal fue la falta de respuesta al tubo de toracostomía de drenaje (40\%). La etiología principal es el empiema pleural tuberculoso. Es un procedimiento ideal para pacientes severamente comprometidos e inmunosuprimidos. Las complicaciones postoperatorias fueron $11.86 \%$. El porcentaje de reoperaciones fue de $3.4 \%$. No se encontró mortalidad asociada al procedimiento. (Rev Med Hered 2002; 13: 90-98).

PALABRAS CLAVE: Ventana torácica, empiema pleural, cirugía, complicaciones.

\section{SUMMARY}

Objective: To describe outcomes of the thoracic window in the treatment of the pleural empyema. Patients and methods: A descriptive, retrospective and traversal study. Subjected patients were included to thoracic window (thoracostomy), between 1990-1998, in the National Hospital Cayetano Heredia. The surgical treatment was studied (technique, reports and complications), morbility and mortality. We studied 59 cases of thoracic window in patient with chronic empyema. They were 39 (66.1\%) males and $20(33.9 \%)$ women. Mean age was $30.9 \pm 14$ years. Results: According to the etiology, $55.93 \%$ had tuberculous pleural empyema, $28.81 \%$ empyema post lung surgery, $11.86 \%$ empyema post pneumonia and $3.39 \%$ empyema post traumatic. $95 \%$ of the cases were drainaged previously with tube of thoracostomy. The predominant radiological pattern was the hidroneumotorax (58\%). The $64.4 \%$ was intervened under regional anaesthesia. The time operative average was of $57.3 \pm$ 28 minutes. The costal arches dried up with more frequency were the $6^{\circ}-7^{\circ}(25 \%)$. The time average of hospitalization was $38.6 \pm 26.9$ days; the time postoperative average was $8.5 \pm 8.3$ 
days. Of the patients that had a bigger pursuit to 3 months ( 33 patients), we could confirm the closing of the thoracic window in 13 cases and lung reexpansión in 21 cases. Conclusions: The main indication was the answer lack to the tube of drainage thoracostomy (40\%). The main etiology is the tuberculous pleural empyema. It is an ideal procedure for patient severely compromised and immunocompromised patients. The postoperatives complications were $11.86 \%$. The percentage of reoperations was of $3.4 \%$. Mortality associated to the procedure was not reported. (Rev Med Hered 2002; 13: 90-98).

KEY WORDS: thoracic window, pleural empyema, surgery and complications.

\section{INTRODUCCIÓN}

La ventana torácica o toracostomía, surge por primera vez, al ser realizado por Elloesser en 1935 quien describe una técnica para drenaje abierto del empiema tuberculoso en casos donde el pulmón no había sido resecado (1). Claggett y Geraci describieron un método de drenaje abierto en empiema post-neumonectomía, con el objeto de evitar la deformante toracoplastía a lo Scheder (2). Ellos resecaban una costilla y dejaban la herida abierta para su irrigación diaria con solución de Neomicina al $0.25 \%$ hasta que ocurriera la esterilización de la cavidad, el cierre de la toracostomía se realizaba en un estadio posterior. Vikkula y Konstiainen describen un método similar, pero creando una ventana de mayor tamaño, resecando 2 ó 3 arcos costales (3). En muchos de sus casos, la ventana se dejó permanentemente abierta debido a la fístula broncopleural u otros factores que no permitieron el cierre. Posteriormente, en 1986, Weissberg considera esta técnica de utilidad en pacientes con empiema crónico sin ser necesariamente neumonectomizados, con o sin fístula broncopleural, considerándola inclusive de gran utilidad para los primeros (4). A diferencia de sus predecesores dejaba la ventana torácica abierta sin cierre quirúrgico posterior.

La ventana torácica se considera como alternativa en casos del empiema pleural que no pueden ser controlados por drenaje cerrado o decorticación, por ejemplo: el empiema postneumonectomía, el empiema temprano recurrente post-decorticación; en las cuales la superficie pleural no se deja intacta y en casos desesperados del empiema micótico o en los pacientes con empiema tuberculoso (1). En otras situaciones, principalmente en pacientes ancianos con empiema crónico, la condición del paciente no permite que la decorticación sea factible (5).

Esta técnica quirúrgica consiste en una pequeña incisión de toracotomía con resección de pequeños fragmentos de una o más costillas dejando la pleura parietal con una amplia comunicación con el exterior, lo que permite un mejor lavado de la cavidad pleural y drenaje. Se ha definido al empiema pleural como "pus en la cavidad pleural" (6). El cúmulo de material purulento puede estar localizado o bien abarcar toda la cavidad pleural. La Sociedad Norteamericana de Enfermedades de Tórax ha clasificado al empiema en 3 fases sobre la base de su evolución intrínseca, y este esquema es útil para planear el tratamiento. La primera fase, es exudativa y se caracteriza por líquido pleural acuoso con pocos leucocitos. Si no se inicia el tratamiento, el empiema evoluciona rápidamente hasta llegar a la etapa fibrinopurulenta de transición (segunda fase), que se caracteriza mas por líquido turbio y espeso, y la aparición de fibrina en superficies pleurales. Al organizarse el empiema, el pulmón comienza a quedar atrapado por una corteza restrictiva de fibrina, iniciándose la fase crónica (tercera fase). La corteza señalada se organiza, y dentro de ella comienzan a proliferar capilares y fibroblastos. En esta fase el pulmón y la parte interna del tórax están cubiertos por una corteza gruesa y limitante del tejido fibroso y fijos $(7,8)$. La fase de organización o crónica puede comenzar 7 a 10 días después del inicio de enfermedad. El orden cronológico de estos fenómenos varía con los microorganismos que originen el empiema pero por la cuarta o sexta semana el proceso ha llegado a la fase crónica (6).

Hay una gran variedad de modalidades terapéuticas para empiema pleural, existiendo aún controversias de todo lo agresivo que debe ser el tratamiento del empiema torácico, algunos autores se inclinan a utilizar como manejo inicial el tubo de toracostomía cerrado, mientras otros apoyan el drenaje operatorio temprano (9). En nuestra institución se estila lo primero, es 
decir, ante la falta de resolución del empiema frente al tubo de toracostomía de drenaje, y la persistencia de fístula broncopleural con o sin succión, inclusive los casos post quirúrgicos, se recurre a la solución quirúrgica mediante la ventana torácica. El cuidado post operatorio Ventana torácica y empiema en el hospital es por 48 a 72 horas seguidas luego por consulta ambulatoria. El paciente y los familiares asumen los cuidados de la herida desde el segundo día post operatorio.

El objetivo del presente estudio fue describir los resultados de la ventana torácica en el manejo del empiema pleural.

\section{PACIENTES Y MÉTODOS}

Es un estudio de tipo descriptivo, retrospectivo y transversal, en que se analizaron los casos de 59 pacientes adultos (mayores de 15 años) intervenidos con Ventana Torácica (toracostomía) por el servicio de Cirugía Torácica y Cardiovascular del Hospital Nacional Cayetano Heredia entre enero de 1990 hasta mayo de 1998.

De los 59 pacientes, 39 (66.1\%) fueron varones y 20 (33.9\%) mujeres. El rango de edades, fue de 15 a 69 años, (media de $30.9 \pm 14$ ), con una mayor frecuencia en el grupo de15 a 24 años $(44.0 \%)$.

Descripción de la técnica quirúrgica de la ventana torácica (Toracostomía):

El procedimiento puede ser realizado bajo anestesia general, regional o aún local, dependiendo del estado general del pacientes y grado de colaboración. La técnica empleada es como sigue:

1. Localización de la zona empiemática de mayor declive mediante punción y aspiración percutánea por toracocentesis.

2. Incisión de piel con bisturí (forma de ojal, generalmente en área donde estaba el dren de toracostomía).

3. Disección por planos hasta arcos costales del espacio intercostal elegido.

4. Disección de periostio, resección de segmento de 5 a 7 cm de arco costal (generalmente 1 ó 2).

5. Se abre la pleura parietal, aspirando la cavidad empiemática y lavando con solución salina.

6. Se revisa hemostasia, para finalmente fijar la pleura a piel con puntos separados con sutura absorvible (poliglactina).

Los pacientes se agruparon de acuerdo a la etiología del empiema :

A.Empiema pleural tuberculoso

B. Empiema post cirugía pulmonary

C.Empiema post neumónico

D. Empiema secundario a traumatismos torácicos Se describen los resultados quirúrgicos, el seguimiento (especialmente en cuanto a cierre espontáneo de ventana torácica y grado de reexpansión pulmonar) y las complicaciones. Las complicaciones postoperatorias fueron clasificadas en tempranas y tardías. Las tempranas que se presentan antes del día 15 del postoperatorio y las tardías que se presentan a partir del día 15 postoperatorio.

\section{RESULTADOS}

Los 59 pacientes fueron agrupados según la etiología del empiema (Tabla $\left.\mathrm{N}^{\circ} 1\right)$ :

1. Empiema pleural tuberculoso: Lo constituyeron 33 casos $(55.93 \%)$, de los cuales, 5 tuvieron TBC multirresistente y 2 TBC multisistémico. Todos los pacientes se encontraban bajo tratamiento específico.

2. Empiema post cirugía pulmonar: Lo constituyeron 17 casos (28.81\%). Este grupo se dividió a su vez en 2 subgrupos: Resección pulmonar (Neumonectomías y 
Lobectomías) y otras cirugías pulmonares. Nueve casos correspondieron a resección pulmonar, de estos 2 fueron neumonectomías (por TBC pulmonar multidrogorresistente) y 7 fueron lobectomías ( 3 casos por hemoptisis masiva por micetoma, 2 por TBC pulmonar activa y 2 por bronquiectasias infectadas). Los 8 casos restantes correspondieron a otras cirugías pulmonares (4 pacientes asociados a rafía de fístula broncopleural y 4 por complicación de quiste hidatídico pulmonar).

3. Empiema post neumónico: Siete casos $(11.86 \%)$ presentaron empiema post-neumónico crónico.

4. Empiema secundario a traumatismos torácicos: Se registraron 2 casos (3.39\%) por traumatismos por arma de fuego al nivel de la región tóracoabdominal. Ambas cirugías tuvieron asociación con lesión diafragmática.

Sólo en 48 historias se especificó la indicación de ventana torácica, de los cuales 19 pacientes (40\%) tuvieron como indicación la persistencia de pus asociada a la falta de reexpansión pulmonar, en segundo lugar 15 casos $(31 \%)$ con indicación sólo de persistencia de pus. En tercer lugar 7 casos $(14.5 \%)$ tuvieron como indicación directa la presencia de empiema tabicado (Tabla No2 ).

En cuanto a los patrones radiológicos, se encontró como principal al hidroneumotórax (34 pacientes, 58.0\%), seguido por el derrame pleural e hidroneumotórax tabicado (ambos con 9 pacientes, $15.0 \%$ ), (Tabla $\mathrm{N}^{\circ} 3$ ).

De los 59 pacientes, $38(64.4 \%)$ fueron intervenidos bajo anestesia regional ( 3 pacientes por anestesia raquídea y 35 por epidural). Un segundo grupo con 20 pacientes (33.90\%), estuvo bajo anestesia general. Y un paciente $(1.7 \%)$ necesitó sólo anestesia local.

El tiempo promedio operatorio fue de $57.3 \pm 28$ minutos, un máximo de 150 minutos y un mínimo de 20 minutos.

Los arcos costales resecados con más frecuencia fueron el $6^{\circ}-7^{\circ}(25 \%)$, seguido por el $5^{\circ}-6^{\circ}$ $(17 \%)$.

De los 59 pacientes, $29(49.15 \%)$ presentaron fístula broncopleural previa a la ventana torácica. Se encontró fístula broncopleural en todas las etiologías de empiema pleural, excepto en el grupo de empiema secundario a traumatismos torácicos (Tabla $\left.\mathrm{N}^{\mathrm{o}} 4\right)$.

Se evidenció que en los 59 pacientes incluidos en el estudio la mayoría fueron drenados con tubo de toracostomía previo a la ventana torácica, excepto en 3 casos en donde se consideró indicado realizar directamente la ventana torácica, debido a empiema tabicado en 2 pacientes y por absceso de partes blandas en el tercer paciente. Los 56 pacientes restantes $(95 \%)$, fueron tratados con tubo previo a la ventana torácica por un periodo de 3 a 90 días, con un promedio de $29.1 \pm 16.5$ días; siendo la mayoría drenados por un tiempo prolongado ya que sólo hubieron 8 pacientes con tubo de toracostomía menor a los 15 días, predominando el grupo de 15-30 días con 29 pacientes $(49.15 \%)$.

Siete pacientes $(11.86 \%)$, presentaron complicaciones postoperatorias, 4 de aparición tardía (los 4 presentaron infección de ventana torácica) y 3 de aparición temprana (2 presentaron abscesos de partes blandas y el tercero presentó neumonía intra hospitalaria). De estos 7 pacientes en 6 de ellos se puede considerar como sobre infección agregada (4 con infección de ventana torácica y 2 con abscesos de partes blandas).

El tiempo de hospitalización tuvo una media de $38.6 \pm 26.9$ días. La mayoría de los pacientes estuvieron hospitalizados de 15 a 60 días. El tiempo de hospitalización postoperatorio tuvo una media de $8.5 \pm 8.3$.

De los 59 pacientes registrados sólo 33 tuvieron controles por más de 3 meses con seguimiento por consultorio externo. 
De estos 33 pacientes, en 13 de ellos se pudo confirmar el cierre de la ventana torácica durante el seguimiento. De los 13 pacientes, 9 presentaron el cierre de la ventana torácica en un tiempo menor o igual a 9 meses y los 4 restantes los hicieron en un tiempo mayor a 9 meses. El promedio de tiempo de cierre de ventana torácica del conjunto es de $7.9 \pm 5.0$ meses, el menor tiempo de cierre fue de 2.6 meses y el mayor fue de 19 meses. Cinco de los pacientes que lograron cierre de ventana torácica espontánea lo hicieron a pesar de la presencia de fístula broncopleural. Las etiologías que se pudieron constatar en el cierre de ventana torácica fueron: TBC pleural en 5 pacientes (38.46\%), otras cirugías pulmonares en 3 pacientes $(23.10 \%)$, resección pulmonar en 3 pacientes $(23.10 \%)$, post neumónico en 1 paciente $(7.7 \%)$ y traumatismo en 1 paciente $(7.7 \%)$.

De los 33 pacientes, $21(63.6 \%)$ presentaron reexpansión pulmonar; en 18 pacientes la reexpansión fue total y 3 parcial. El tiempo promedio de reexpansión pulmonar fue de $5.29 \pm$ 4.21 meses, con un rango de 2.23 a 17.70 meses.

De los 18 pacientes con reexpansión pulmonar total, la etiología del empiema fue: TBC pleural en 9 casos (50\%), otras cirugías pulmonares en 3 casos $(16.67 \%)$, post neumónico en 3 casos (16.67\%), resección pulmonar en 2 casos $(11.1 \%)$ y traumatismo en 1 caso $(5.56 \%)$. Se encontró que en 14 de ellos hubo fístula broncopleural previa a la ventana torácica que se resolvieron en el postoperatorio sin la necesidad de ningún procedimiento adicional. Los 4 pacientes restantes no presentaron FBP.

De los 3 pacientes con reexpansión pulmonar parcial, 2 pacientes $(66.67 \%)$ presentaron como etiología del empiema resección pulmonar y 1 paciente $(33.33 \%)$ presentó TBC pleural multidrogorresistente. Los 3 casos con reexpansión parcial presentaron fístula broncopleural previa a la ventana torácica, uno de los casos con FBP se resolvió espontáneamente los otros 2 requirieron procedimientos adicionales.

Dos fueron los pacientes que no lograron reexpansión hasta el momento de su último control radiológico un caso correspondió a empiema post neumónico de neumonía extrahospitalaria con antecedente de TBC pulmonar secuelar y el otro a TBC pleuropulmonar multirresistente. Los 2 casos presentaron fístula broncopleural previa a la ventana torácica que fueron resueltos por procedimientos adicionales.

Dos pacientes (3.4\%), fueron reoperados posterior a la ventana torácica, a los cuales se les realizó toracoplastía. Un paciente con empiema TBC pleuropulmonar multirresistente, el segundo paciente con empiema post resección pulmonar complicado con infección de sutura de fístula broncopleural.

No se encontró mortalidad asociada a ventana torácica.

\section{DISCUSIÓN}

La ventana torácica ofrece una alternativa de solución al empiema pleural, debido a que la amplia abertura permite limpieza diaria de la cavidad, aunque la mayoría de los investigadores han utilizado la ventana torácica sólo como tratamiento de empiema posterior a neumonectomía, en nuestro hospital se utiliza, además, sin que necesariamente haya resección previa.

Los pacientes sometidos a ventana torácica en nuestro hospital presentan un promedio de edad similar al reportado en otras series. Aunque a diferencia de ellas, en la nuestra, se observa un ligero predominio en pacientes más jóvenes, que corresponden a los infectados con tuberculosis, esto relacionado a la alta prevalencia en nuestro medio (10). Además, en 
este estudio no se presentaron pacientes con neoplasias que generalmente ocurren a mayor edad.

En nuestra serie, los pacientes que fueron sometidos a ventana torácica tuvieron como indicación principal la falta de respuesta adecuada ante el tubo de drenaje cerrado, es decir, la persistencia de drenaje purulento y falta de reexpansión pulmonar en un periodo de 2 a 3 semanas. Hallándose en algunos casos a pesar del uso de succión intermitente y/o continua, la persistencia del colapso pulmonar, presentando éstos, como es de suponer mayor tiempo con tubo cerrado. La ventana torácica en la literatura, está indicada en pacientes que presentan empiema pleural con o sin fístula broncopleural que no respondieron al tratamiento antibiótico y tubo de drenaje $(5,11,12)$; también está indicada de primera intención como alternativa frente al tubo de drenaje cerrado, para obtener un drenaje adecuado especialmente cuando el contenido del empiema es espeso, grueso y con tejido fibrótico $(12,13)$; o en aquellos pacientes en que se considera que no tendrán buena respuesta al tratamiento médico, conservador y/o decorticación; tales como el empiema post-neumonectomía, empiema recurrente temprano posterior a la decorticación, en los cuales no se deja superficie pleural intacta, y en casos desesperados de empiema micótico (14). Igualmente, la ventana torácica, es necesaria para evacuar el contenido de la cavidad pleural cuando hay síntomas de complicación por fístula broncopleural con manifestaciones sistémicas que no responden al manejo antibiótico o a la irrigación (11). La ventana torácica es también usada donde el tubo de drenaje intercostal cerrado debe ser alto e incómodo por ejemplo en zonas altas de la axila o en el área paravertebral. En la literatura no existen referencias que puntualicen en qué casos se debe someter el paciente a ventana torácica sin necesidad de tubo de drenaje cerrado previo, los autores sólo mencionan la necesidad de tratamiento más agresivo en casos de empiema quirúrgico sobre todo en post-neumonectomizados, en TBC mixta (en que está implicada la presencia de fístula broncopleural), debido al mal estado general del paciente, tales como ancianos, desnutridos, inmunosuprimidos y enfermedades subyacentes que debilitan a los pacientes y de alto riesgo quirúrgico; todos los anteriores son considerados candidatos a ventana torácica por ser de menor riesgo frente a otra técnicas quirúrgicas (15).

Dentro de la etiología del empiema en nuestro estudio, predomina el empiema pleural tuberculoso, a diferencia de lo observado en otras series, en las que se presenta como principal causa al empiema post-neumónico. Ali and Unruh (16) presentan en su serie más del $50 \%$ de pacientes con empiema post-neumónico. En décadas pasadas el empiema pleural a menudo era relacionado con la TBC pulmonar, pero, debido a los avances en la terapia de drogas anti-TBC, la incidencia de tuberculosis ha declinado gradualmente en el ámbito mundial, ocurriendo la pleuresía TBC sólo en pacientes con inmunosupresión y en zonas endémicas, generalmente en países en vías de desarrollo como el nuestro. Es entonces, que hoy en día la mayoría de casos de empiema es ocasionada como complicación de neumonía o de operaciones de cáncer pulmonar o enfermedad abdominal $(11,17,18)$. La predominancia del empiema crónico por tuberculosis, se explica por la alta incidencia de TBC pulmonar en nuestra población atendida. Además, se sabe que en la TBC se producen frecuentemente lesiones tales como pleuresía recurrente, ruptura de una lesión necrótica en la cavidad pleural o complicación de un neumotórax artificial contiguo que son causa frecuente de empiema crónico en pacientes con TBC.

La segunda causa más frecuente de empiema pleural reportado en la literatura es la infección pleural postquirúrgica posterior a cirugía del esófago, pulmón o mediastino $(19,20)$. La incidencia de empiema postneumonectomía es de 2.2 a $23 \%(21,22)$. Ueda y colaboradores, refieren que el piotórax ocurre en el 11.4\% de las neumonectomías (19) y puede ocurrir en 1 a $3 \%$ de los pacientes post lobectomía (6). Esto coincide con nuestra serie donde la segunda causa de empiema pleural es la post quirúrgica. La incidencia de empiema después de resección pulmonar varía con la indicación de la resección (inflamatorio o neoplásico, con o sin radiación preoperatoria). Lynn (23) en pacientes con TBC pulmonar con examen de esputo positivo reporta $6.7 \%$ de casos de empiema con fístula broncopleural post-lobectomía, 
en tanto Teixera reporta un $10 \%$ de casos de empiema post-lobectomía en pacientes con examen de esputo positivo $(19,24)$. En concordancia con estos autores la mayoría de nuestros casos por resección son debido a lesiones de TBC activas. En el empiema postneumonectomía, Enrola y Vikkula reportan que usualmente se manifiesta en las 2 a 4 semanas del post-operatorio (21); en le serie de Goldstraw se reporta un tiempo de 5 semanas (25) y en la serie de Lemmer, 4 semanas (26). En nuestro estudio los 2 casos con empiema post-neumonectomía la aparición del empiema ocurrieron dentro de las 2 semanas luego de la cirugía.

La fístula broncopleural en nuestra serie se presentó en mayor porcentaje en los pacientes con TBC pleural, a diferencia de lo reportado en la literatura en la que el grupo de mayor riesgo de presentar fístula broncopleural son los pacientes con empiema post quirúrgico sobre todo los pacientes con empiema postneumonectomía (4). La fístula broncopleural postneumonectomía representa la más temida complicación en la cirugía torácica, siendo el diagnóstico y el manejo terapéutico el mayor problema. Esta es producida por la dehiscencia del muñón bronquial y lleva al empiema por contaminación e infección retrógrada del árbol bronquial remanente por vía de la fístula (27). Enrola y Vikkula reportan la presencia de fístula broncopleural en pacientes postneumonectomizados en el $33 \%$ de los casos $(3,21)$, además la fístula broncopleural, es más común en la post-neumonectomía que en la lobectomía (21). La fístula broncopleural postneumonectomía ocurre más frecuentemente en la Ventana torácica y empiema, 2002 resección por TBC debido a la presencia de TBC activa oculta en el muñón bronquial generalmente presente en el 5 a $10 \%$ de resecciones (28). El éxito del tratamiento, al menos inicialmente depende de un drenaje adecuado, lo que en la practica supone la realización de la ventana torácica algunas veces precedida por tubo de toracostomía (29). El drenaje temprano en un empiema con fístula broncopleural es mandatorio para prevenir la sepsis y la aspiración (28).

La incidencia y desarrollo de la fístula broncopleural, en el empiema post-neumonectomía está reportado entre los rangos de: 2 a 13\% (21,30). Es raro observar empiemas sin fístula broncopleural por lo que se considera que la fístula broncopleural es la principal causa de empiema post-neumonectomía, viéndose inclusive que una vez cerrada la fístula no hay recurrencia del empiema. (12,27,31). Puede lograrse cierre espontáneo de fístula broncopleural luego de la creación de la ventana torácica reportado por Adebo (32) en 3 de 4 casos. Weissberg (4) lo reporta en sus 7 casos con obliteración completa en 24 meses.

Más de la mitad de los casos fueron sometidos a ventana torácica bajo anestesia regional. La ventaja de este tipo de anestesia estriba que es de menor riesgo para el paciente en cuanto efectos adversos y necesidad de monitorización estricta de funciones vitales, aunado al menor costo y tiempo operatorio, así como tiempo de recuperación. También como se ha reportado en la literatura latinoamericana se hizo uso de la anestesia local en un paciente muy comprometido, así como Leone (5) reporta en sus 16 casos, de los cuales 6 eran postquirúrgicos.

El tiempo operatorio es relativamente corto, lo que constituye una gran ventaja, pues al disminuir el tiempo de exposición de la región torácica a operar se minimiza el riesgo de infección operatoria.

Existen pequeñas diferencias en el cuidado postoperatorio según la series. Así tenemos que según Smolle-Juttner (14), en los 3-4 primeros días los apósitos se cambiaron bajo anestesia de acción corta tal como el propofol, en los días subsiguientes no se requirió uso de ningún medicamento sedante o analgésico. En el método de Clagett indicado para el empiema postneumonectomía se realizan irrigaciones diarias del espacio pleural con solución antiséptica, más frecuentemente de 1:20,000 de chlorhexide gluconate (Hibitane), iniciando a las 24 horas de la toracostomía para lograr la esterilización del espacio pleural (33). En la serie argentina, Leone reporta el uso de azúcar para evitar el cierre prematuro de la ventana torácica logrando controlar la infección en la segunda a tercera semana post-operatoria y granulación en la mayoría de los casos al sexto mes (5). Otros consideran el uso de tampones con apósitos con solución salina isotónica 2 veces por día y luego una vez por día (21). En nuestra serie se 
realizó irrigación con cloruro de sodio al $0.9 \%$ a partir del primer día post-operatorio no requiriendo sedación ni analgesia por parte del paciente, enseñando la técnica de curación a la familia para los cuidados posteriores.

No se reportan complicaciones propias de la ventana torácica, sin embargo hemos considerado 7 casos de complicación post-ventana torácica, 6 de ellos podrían ser considerados como sobreinfección agregada; 2 de los cuales fueron los abscesos de partes blandas de aparición temprana (dentro de la primera semana) y 4 la llamada ventana torácica infectada (presentes en los primeros días o meses siguientes). Es quizá la presencia de fístula broncopleural que predispuso a la sobreinfección y que complicó el cuadro en esta ocasión cuya evolución siempre es de desarrollo impredecible como lo menciona Vikkula, sobre todo en pacientes post neumonectomizados (3).

El tiempo de hospitalización promedio de 38,6 días fue similar al reportado por Simmons en pacientes con empiema torácico sometidos a ventana torácica (34). Encontrándose en la literatura que el tiempo de hospitalización fue ligeramente mayor para los pacientes con empiema sometido a otros tratamientos diferentes a ventana torácica, (35). Esto se corrobora en nuestra serie en la que la mayoría de pacientes son sometidos a otros procedimientos antes de la realización de la ventana torácica, lo que explicaría la demora en su realización o la toma de decisión de indicarla. Por otro lado el tiempo post-operatorio es corto, en nuestra serie presentó una media de 8.54 días, pues los cuidados post-ventana torácica son sencillos y es mínima la presencia de complicaciones post-operatorias, reportándose casos de alta al día siguiente de la operación. Vemos entonces que una vez indicada la ventana torácica el tiempo hospitalario sería menor, por lo que una pronta elección de ella, evitaría una estadía prolongada del paciente minimizando costos y riesgos de sobreinfección por gérmenes intrahospitalarios.

En nuestra institución se espera el cierre espontáneo de la ventana torácica, no se realizó en ningún caso cierre secundario. Se encontró un promedio de cierre de ventan torácica de 7.94 meses. Otros autores reportan resultados similares, principalmente Weissberg (4) con un promedio de cierre de 6 meses en 10 de 12 pacientes, los 2 pacientes restantes tuvieron un tiempo de cierre de 8 y 24 meses. Smolle-Juttner reporta 4 pacientes con cierre espontáneo en un tiempo promedio de 13.2 meses (14).

Tuvimos casos que presentaron reexpansión pulmonar total a pesar de presentar fístula broncopleural en empiema post-neumonectomía en la que había fallado la succión con tubo de drenaje cerrado, con cierre espontáneo de fístula broncopleural; aunque no hemos podido hacer diferencia de los casos según el tamaño de fístula. Ali y colaboradores hallan que paradójicamente el factor que más comúnmente indicaba reexpansión potencial era el colapso total del pulmón contra el mediastino ya que esto indicaba que la pleura no presentaba fibrosis (36). Un parénquima que no muestra cavitación ni bronquiectasia potencialmente puede reexpanderse. La presencia de cavitación y fibrosis a menudo con adhesión disminuyen las probabilidades de reexpansión. Un pulmón fibrótico que no se colapse totalmente y con pleura engrosada probablemente no responda al tratamiento. Algunos de nuestros casos presentaron tubo de drenaje intercostal por mas de un mes sin reexpansión que luego con ventana torácica reexpandieron. Hoy en día el drenaje abierto se instituye en un estadio mas temprano, previo al mes antes de observarse la ausencia de reexpansión con succión de drenaje, asegurando que no se presente el cambio mediastinal.

Se halló un gran número de casos de pacientes con empiema asociado a fístula broncopleural ya sea en pacientes que lograron la reexpansión pulmonar total o en los que hubo sólo reexpansión parcial. Señalando aparentemente que la fístula broncopleural no es un factor limitante para la reexpansión pulmonar posterior a la ventana.

En conclusión, la ventana torácica es un procedimiento quirúrgico que presenta una mortalidad muy baja, en nuestra serie no se reportó mortalidad, lo que concuerda con lo encontrado en otras series (37).

\section{REFERENCIAS BIBLIOGRAFICAS}

1.Elloesser L. An operation for tuberculous empyema. Surg Gynecol Obstet 1935; 60:1096-7

2.Clagget OT; Geraci JE. A procedure for the management of Post-Neumonectomy 
empyema. J Thorac Cardiovasc Surg 1963; 45:141-145.

3.Vikkula L, Konstiainen S. Postneumonectomy empyema in pulmonary carcinoma patients. Scand J Thorac Cardiovasc Surg 1970; 4:267-7

4.Weissberg D. Empyema and bronchopleural fistula. Experience with Open Window Thoracostomy. Chest 1982; 4:47-50.

5.Leone RR, Torres AD, Salgado R. Ventana torácica en el tratamiento del empiema pleural crónico. Rev Arg Cirug 1988; 55:132-4

6.Shield TW. Parapneumonic Empyema. En General Thoracic Surgery-44th Ed. Hood, RM. Williams \& Wilkins.Baltimore.1994. pp: 684-711.

7.American Thoracic Society: A Statement of Subcommittee of Surgery Management of Nontuberculous Empyema. Am Rev Resp Disease 1962; 85:925-6

8.Hood, R. Maurice.Thoracic Surgery-2nd ED-Philadelphia: Lea and Febiger. 1993.

9.Smith JA, Mullerworth MH, Westlake GW, Tatoulis J. Empyema Thoracis: 14 year experience in a teaching center. Ann Thorac Surg 1991; 59:39.

10.Dirección general de Salud de las personas. Programa Nacional de Control de la Tuberculosis. Tuberculosis en el Perú. Informe 97. Lima, Perú.

11.Lioka S, Sawamura K, Mori T, Iucchi K, Nakamura K., Monden Y., Kawashima Y. Surgical Treatment Of Chronic Empyema. A new one stage treatment. J Thorac Cardiovasc Surg 1985; 90:179-85.

12.Shamji FM, Ginsberg RJ, Cooper JD, Spratt EH; Goldberg M, Waters PF, Ilves R, Todd TR, Pearson FG. Open window thoracostomy in the management of pneumonectomy Empyema with or without bronchopleural fistula. J Thorac Cardiovasc Surg 1983; 86:812-22

13.Samson, PC. Empyema Thoracis. Essentials of present day management. Ann Thorac Surg 1971; 11(3):210-222.

14.Smolle Juttner F, Beuster W, Pinter H, Pierer G, Pongratz M, Friehs G. Open window thoracostomy in pleural empyema. Eur J Cardiothorac1992; 6(12):635-8.

15.Bussetti JH, Barato E, Iwabuchi U, Mayo SV, Alfabet C, Isola A. Commentaries sobre a cirugía de Elloesser. Commentaries about Elloesser surgery empyema. Arq Med ABC 1991; 14(2):55-8.

16.Ali I, Unrun H. Management of empyema thoracys. Ann Thorac Surg 1990; 50:355-9.

17.Le Blanc KA, Tucker WY. Empyema of the thorax. Ginecologic Obstetric 1984; 158: 6670 .

18. Sherman MM, Subramanian V, Berger RL. Management of thoracic empyema. Am J Surg 1977; 133:474-9.

19.Ueda H, Shibata K, Kusano T. Post operative piotórax. Surgery Today. The Japanese Journal of Surgery 1992; 2: 115-9.

20.Snider GL, Saleb SS. Empyema of the Thorax in Adults: Review of 105 Cases. Chest $1968 ; 54: 12$.

21.Eerola S, Virkkula L. and Varstela E. Treatment of postneumonectomy empyema and associated bronchopleural fistula. Scand Journal Thorac Cardiovasc 1988; 22:235-38. 22.Pecora DV. Pneumonectomy for pulmonary tuberculosis. Dis Chest 1965; 48:153.

23.Light RW. Management of Paraneumonic effusions. Am J Med 1980; 69:507.

24.Teixera J. The present status of thoracic surgery in tuberculosis. Dis Chest 1968; 53:19.

25.Goldstraw P. Treatment of postneumonectomy empyema. The cause of fenestration. Thorax 1979; 34:740- 745 .

26.Lemmer MJ, Botham JH, Orringer MB. Modern management of adult thoracic empyema. JThorac Cardiovasc Surg1985; 90: 849-55.

27.Hurtvitz RJ, Tucker BL.The Elloesser flap: past and present. J Thorac Cardiovasc Surg 1986; 92:958-64.

28.Dorman JP, Campbell D, Grover FL, Trinkle JK. Open thoracostomy drainage of postneumonectomy empyema with bronchopleural fistula. J Thorac Cardiovasc Surg 1973; 66:979-81.

29.Galvin IF, Gibbons JR, Maghout MH. Bronchopleural Fistula. A novel type of open window thoracostomy. J Thorac Cardiovasc Surg.1988; 96:3433-5.

30.Nagasaki F, Fleinger BJ, Martini N. Complications of surgery in the treatment of the 
carcinoma of the lung. Chest 82:25-9.

31.Adler RH, Plant ME. Post-pneumonectomy empyema. Surgery 1977; 71:210.

32. Adebo OA, Osinowa O. Management of empyema and bronchopleural fistula, Experience With Elloesser window. Trop Doc 1987; 17: 26-29.

33.Bayes AJ, Wilson JA, Chin RC, Errett LE, Hedderich G, Munro D. Claggett Open window thoracostomy in patients who had and had not undergone pneumonectomy. The Canadian Journal Of Surgery 1987; 30:329- 32.

34.Simmons EM, Sauer P, Elkadi A, MacKenzie JW, Almond CH. Review of Non Tuberculous Empyema at the University of Missouri Medical Center from 1957 To 1971. J Thorac Cardiovasc Surg 1972; 64:578-85.

35. Carcelèn MRI. Empiema pleural bacteriano en adultos. Tesis para Bachiller en Medicina. Universidad Peruana Cayetano Heredia. Lima - Perú 1987.

36.Ali SM, Siddiqui AA, Mc Lauguin.open drainage of massive tuberculous empyema with progressive reexpasion of the lung An old concept revisited. Ann Thorac Surg 1996; 621: 218-223.

37.Carey JA, Hamilton JRL, Spencer DA, Gourld K, Hasan A. Empyema thoracis: A role for open thoracotomy and decortication. Archives of Disease in Childhood 1998; 6:510.

\section{Correspondencia:}

Dr. Enrique Castañeda Saldaña.

Departamento de Cirugía, Universidad Peruana Cayetano Heredia. Av. Honorio Delgado 430, Lima 31, Perú.

Email: ecasta@upch.edu.pe. 